\title{
Solar energetic particle events related to disk-centre full-halo coronal mass ejections
}

\author{
E. Valtonen* \\ Department of Physics and Astronomy, University of Turku, Finland \\ E-mail: eino.valtonen@utu.fi \\ D. Ameri \\ Department of Physics and Astronomy, University of Turku, Finland \\ and \\ Department of Ecology, University of Basrah, Karmat Ali B.P. 49, Basrah, Iraq \\ E-mail: dheyaa.a.ameri@utu.fi
}

\begin{abstract}
We have investigated the occurrence and characteristics of solar energetic particle (SEP) events related to full halo coronal mass ejections (FHCMEs) from source locations close to the central meridian of the Sun. Halo CMEs detected by SOHO/LASCO between 1996 and end of 2014 originating from source locations between solar longitudes E10 and W10 were first identified. The CMEs were divided in three groups according to the latitude of the source region: 26 equatorial events in the latitude range [N10, S10], 16 northern events in the range (N10,N20], and 22 southern events in the range (S10, S20]. We then searched for associated solar proton events based on SOHO/ERNE observations. In altogether 25 cases (13 equatorial, 6 northern, and 6 southern events) an association was found. For these events, we found good correlations of the peak intensities of $2 \mathrm{MeV}, 20 \mathrm{MeV}$, and $68 \mathrm{MeV}$ protons with both the CME speed and the X-ray flare magnitude, as determined by the soft X-ray peak flux or the event integrated flux. The correlation coefficients ranged from 0.47 to 0.83 for the CME speed, from 0.63 to 0.88 for the X-ray peak flux and from 0.64 to 0.89 for the X-ray integrated flux depending on the proton energy.
\end{abstract}

The 34th International Cosmic Ray Conference,

30 July- 6 August, 2015

The Hague, The Netherlands

\footnotetext{
* Speaker.
} 


\section{Introduction}

Solar energetic particles (SEPs) are accelerated in magnetic field reconnection processes related to flares and the launch of coronal mass ejections (CMEs) and by shock waves driven by CMEs in the solar corona and in interplanetary space. SEP events from flare or CME source regions in the western hemisphere of the Sun tend to be most frequent (see, e.g., [1]) due to the favourable magnetic connection for particle propagation from the sources to the observer near Earth. How the peak intensities and energy spectra of SEPs are related to the properties of flares and CMEs is, however, a long-standing open question. If a major part of SEPs is accelerated by a CME-driven shock, then one might expect strong correlation of the SEP peak intensities and CME speeds. On the other hand, if particles are accelerated mainly in flare-related processes, then the flare properties could be expected to be the most important factors determining the particle intensities. Both of these aspects have been widely studied by using various data sets of particle observations and characterisations of coronal mass ejections and solar flares [2, 3, 4, 5, 6, 7, 8, 9, 10]. Usually, the correlation coefficients between the logarithms of proton peak intensities and the logarithms of soft $\mathrm{X}$-ray $(0.1-0.8 \mathrm{~nm})$ flare magnitudes or CME speeds vary broadly in the range 0.4-0.6. These are relatively low values, but significant in the sense that there is a low probability that such values would result from uncorrelated data sets by chance. For more restricted data sets, e.g. in proton energies, source locations, or CME properties, higher values have also been reported (e.g., [11]). In spite of the relatively good correlations, proton intensities associated with X-ray flares with similar magnitudes or with CMEs with similar speeds can vary by orders of magnitude. This indicates that there must also be other factors determining the observed proton peak intensities [4, 12].

We have studied the occurrence and characteristics of solar proton events associated with full halo CMEs launched from close to central meridian of the Sun and the correlations of proton peak intensities at several energies with CME speeds and soft X-ray flare magnitudes.

\section{Data selection}

From the SOHO/LASCO [13] catalog of full halo CMEs (http: //cdaw.gsfc.nasa. gov/CME_list/HALO/halo.html) [14] we selected all events from 1996 till the end of 2014 with the given source locations between solar longitudes E10 and W10. We then divided the selected events in three groups: equatorial events in the latitude range [N10, S10], northern events in the range $(\mathrm{N} 10, \mathrm{~N} 20]$, and southern events in the range (S10, S20]. In the latitude division, the solar B0 angle, the angle between the solar equator and the ecliptic plane, was taken into account, i.e., the event division is based in latitudes as seen from Earth. Altogether 64 full halo CMEs were found, 26 of which belonged to the equatorial, 16 to the northern, and 22 to the southern group. No limitation was set for the speed of the CMEs. The time and latitude distribution of the selected events is presented in Figure 1 (a). SOHO/ERNE [15] proton data at energy channels 1.33-2.66 MeV (nominal energy $2 \mathrm{MeV}$ ), 13.8-29.0 MeV (20 MeV), and 50.8-86.7 MeV (68 MeV) were then inspected to find out solar proton events associated with the selected CMEs. In 25 cases (13 equatorial, 6 northern, and 6 southern events) an association was found at least in one energy channel. In the SOHO/LASCO halo CME catalog, the estimated space speed of the CMEs is also given, and in Figure 1 (b) the space speed of all the CMEs is shown as a function of time. The 


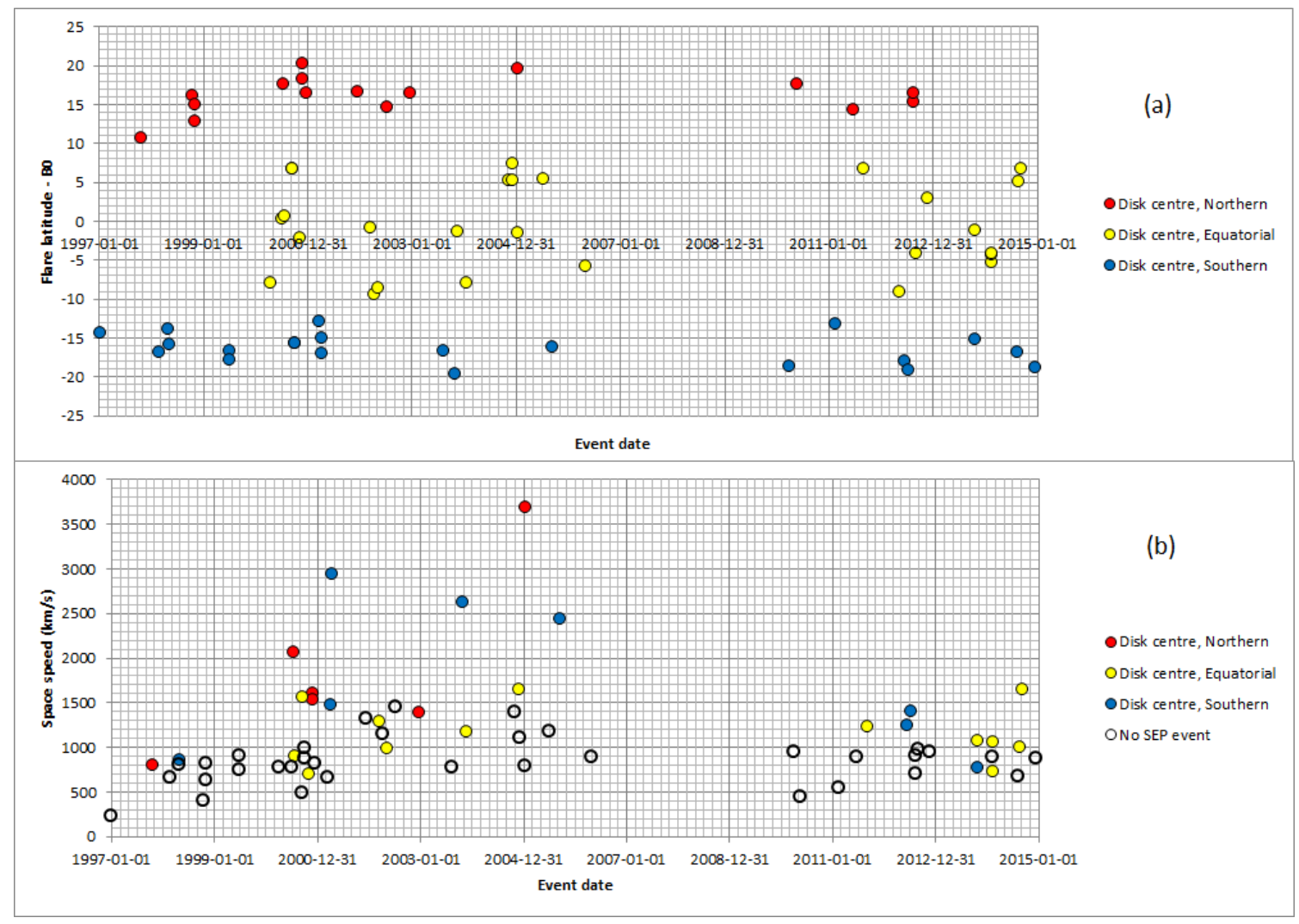

Figure 1: Latitude distribution of selected halo CMEs as function of time (a) and space speeds of selected CMEs (b). In (b), filled symbols indicate events associated with proton events and unfilled circles represent events with no associated SEPs.

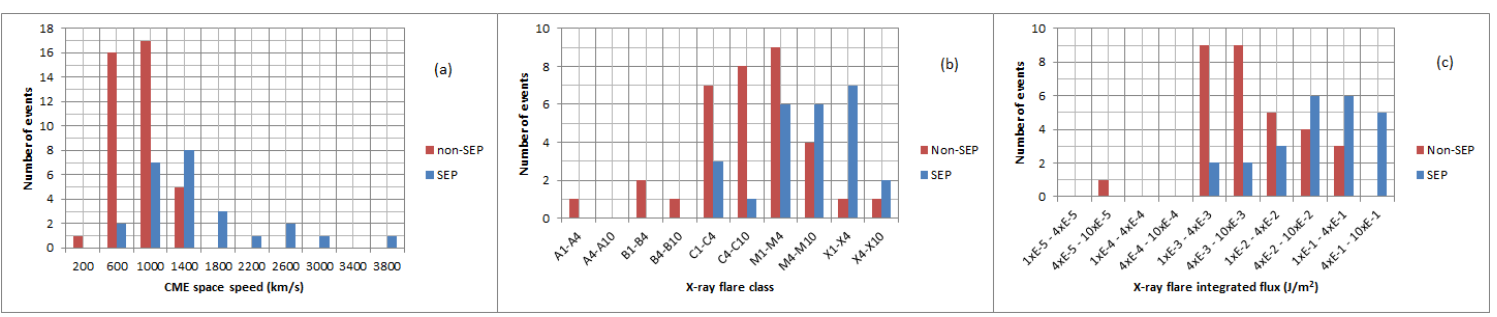

Figure 2: (a) Distribution of space speeds of CMEs associated and not associated with proton events. (b) X-ray flare class distribution of SEP and non-SEP associated events. (c) As (b), but for event integrated $\mathrm{X}$-ray flare flux.

CMEs for which a proton event association was found are presented with filled symbols. Unfilled circles represent CMEs for which no SEP association was found in the ERNE proton data.

The space speed distribution of CMEs associated and not associated with proton events is presented in Figure 2 (a). The minimum and maximum space speeds of the CMEs associated with proton events were $701 \mathrm{~km} / \mathrm{s}$ and $3682 \mathrm{~km} / \mathrm{s}$, respectively, with the average (median) speed of $1530 \mathrm{~km} / \mathrm{s}(1392 \mathrm{~km} / \mathrm{s})$. The average (median) speed of the equatorial CMEs was $1156 \mathrm{~km} / \mathrm{s}$ $(1070 \mathrm{~km} / \mathrm{s})$, while those of the northern and southern events were $1847 \mathrm{~km} / \mathrm{s}(1572 \mathrm{~km} / \mathrm{s})$ and $2026 \mathrm{~km} / \mathrm{s}(1964 \mathrm{~km} / \mathrm{s})$, respectively. The highest-speed CME was among the northern events. The 
average (median) space speed of all selected CMEs was $1121 \mathrm{~km} / \mathrm{s}(941 \mathrm{~km} / \mathrm{s})$ and that of the CMEs not associated with proton events $858 \mathrm{~km} / \mathrm{s}(866 \mathrm{~km} / \mathrm{s})$. Based on the acceleration values given in the halo CME catalog, 15 of the proton event-associated halo CMEs were decelerating and 10 accelerating. X-ray flare class and event integrated X-ray flux distributions for SEP and non-SEP events are shown in Figure 2 (b) and (c), respectively.

Possible inclusion of the SEP events associated with disk-centre halo CMEs in some other SEP lists was examined. It turned out that all but four events from the time period covered by the list [6] were also in this list. With one exception (September 16, 2000 event) all of these proton events missing from the list [6] were seen only in the lowest, $2 \mathrm{MeV}$, proton channel. Although not in the list [6], the September 16, 2000 event is included in the list [16]. In [6] the authors identified an event also on April 15, 2002 and associated it with a source at W79. This event also coincides rather well with a halo CME at 03:50 UT in our list. However, there was a relatively large SEP event going on at this time which started already in the morning of April 14, and we were unable to see any additional enhancement in any of our proton channels which could have been associated with the disk-centre halo CME (or with the source at W79). The list [6] includes also a SEP event on July 15, 2002, which is further discussed in the next section. All of our proton events associated with disk-centre halo CMEs from the period covered by the list [17] are also in this list. The list [17] includes two events which had been associated with our selected disk-centre halo CMEs, but for which we saw no enhancements in the ERNE proton data.

Most (18/25) of the disk-centre halo CMEs associated with proton events were also associated with decametric-hectometric type II radio emission, which has been found to be a good indicator of solar proton events [1, 18]. There were seven exceptions, five of which had low intensities $\left(<10^{-2} \mathrm{~cm}^{-2} \mathrm{~s}^{-1} \mathrm{sr}^{-1} \mathrm{MeV}^{-1}\right)$ or were not observed at all at $20 \mathrm{MeV}$. The remaining two had intensities $>10^{-1} \mathrm{~cm}^{-2} \mathrm{~s}^{-1} \mathrm{sr}^{-1} \mathrm{MeV}^{-1}$. Nine of our non-SEP-associated events, including the events of April 15 and July 15, 2002 mentioned above, have also been reported to be associated with decametric-hectometric type II emissions.

\section{Results}

We have analysed our restricted data set of solar proton events associated with halo CMEs launched from near central meridian of the Sun and investigated the dependence of the proton peak intensities at three energies, $2 \mathrm{MeV}, 20 \mathrm{MeV}$, and $68 \mathrm{MeV}$, on the $\mathrm{CME}$ speeds and on the magnitude of the soft X-ray flares. Because the number of proton events was relatively small (25), and because no significant difference was found between the northern, equatorial, and southern groups, although the equatorial events tended to be associated with lower CME speeds and X-ray peak fluxes (see Figures 3 and 4), all three groups were treated together to maintain reasonable statistics. For analysis,the background levels were subtracted from all peak intensities. Usually, the backgrounds were insignificant, several times lower than the peak intensities. Error bars in proton intensities shown in Figures 3 and 4 take into account, in addition to the statistical errors, also the uncertainties in defining the peak intensities, which were determined by eye close to the end of the usually rapid main rise phase of the event. The error bars correspond to roughly $2-3 \sigma$ statistical errors depending on the event characteristics. 


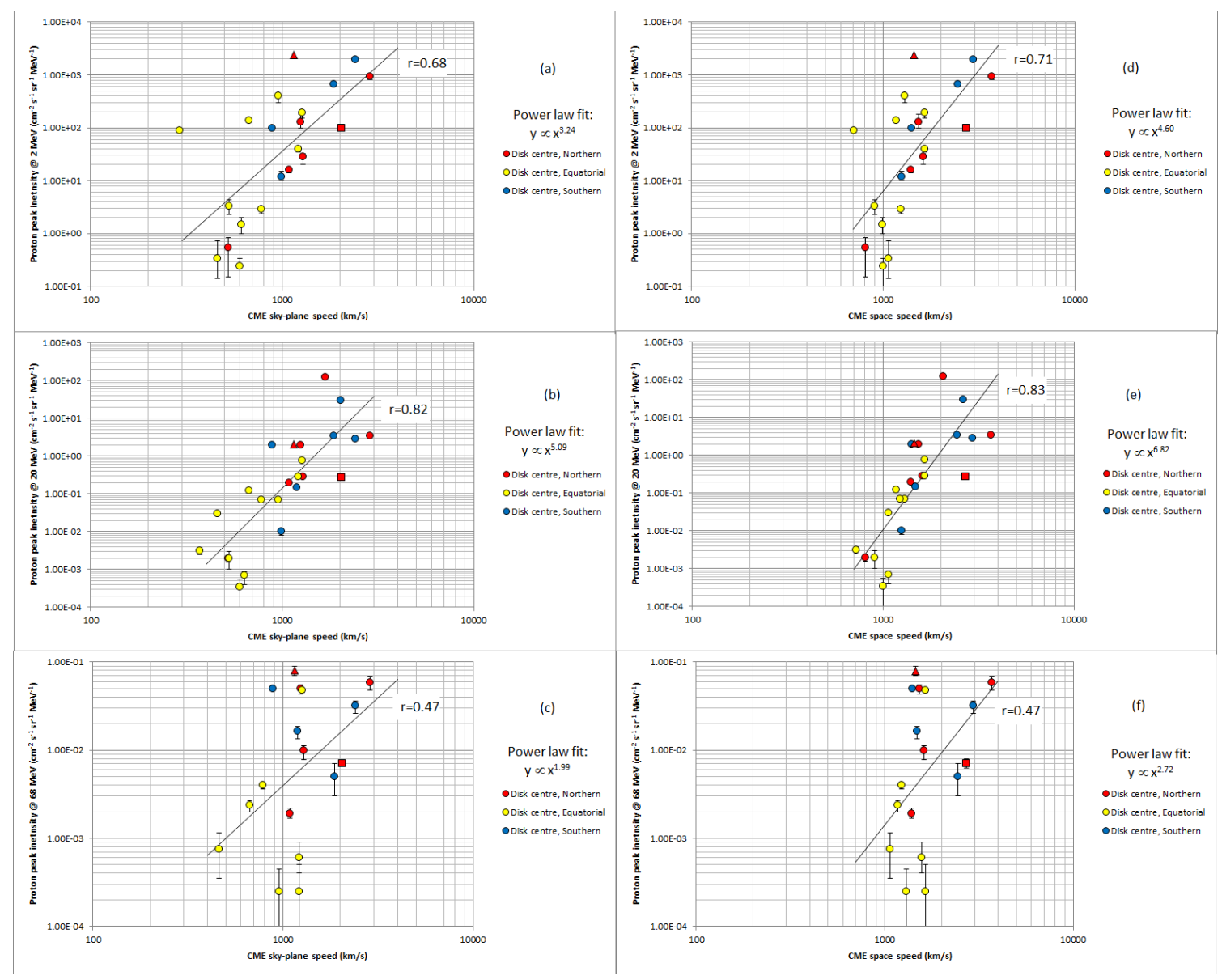

Figure 3: Proton peak intensities at 2, 20, and $68 \mathrm{MeV}$ as function of CME sky-plane speed (a-c) and space speed (e-f).

The main results are presented in Figures 3 and 4. Figure 3 shows the proton peak intensities at 2, 20 and $68 \mathrm{MeV}$ as a function of the sky-plane speed of the CMEs (left-hand panels (a) to (c) of Figure 3) and as a function of the space speed (right-hand panels (d) to (f) of the figure) in logarithmic scales. In both cases, the speeds are those given in the SOHO/LASCO halo CME catalog. The linear correlation coefficients, $r$, are given in the plots. All correlations are significant at $\gtrsim 92 \%$ level with the lowest and least significant correlation at the highest energy. The lowest correlation at $68 \mathrm{MeV}$ can be at least partly explained by the uncertainty in determining the peak intensities at this energy (see the error bars in plots (c) and (f)) and by the lowest number (15) of events. At $20 \mathrm{MeV}$, which can be considered as the most reliable data set (22 events), the correlation is surprisingly high (0.82). This can be compared, e.g., with the result of [10] who for a data set of 34 gradual SEP events observed during transient solar wind conditions obtained the correlation coefficient 0.74 for the dependence between the $20 \mathrm{MeV}$ proton peak intensities and the $\mathrm{CME}$ sky-plane speeds (in log-log scale). In [11] a correlation coefficient of 0.78 was reported for the dependence of the logarithm of the proton peak intensity on the CME speed for the solar central region (E30 - W30) events, which was higher than for eastern or western events. In [11] a higher correlation coefficient was obtained for the peak intensity dependence on the radial CME speed 


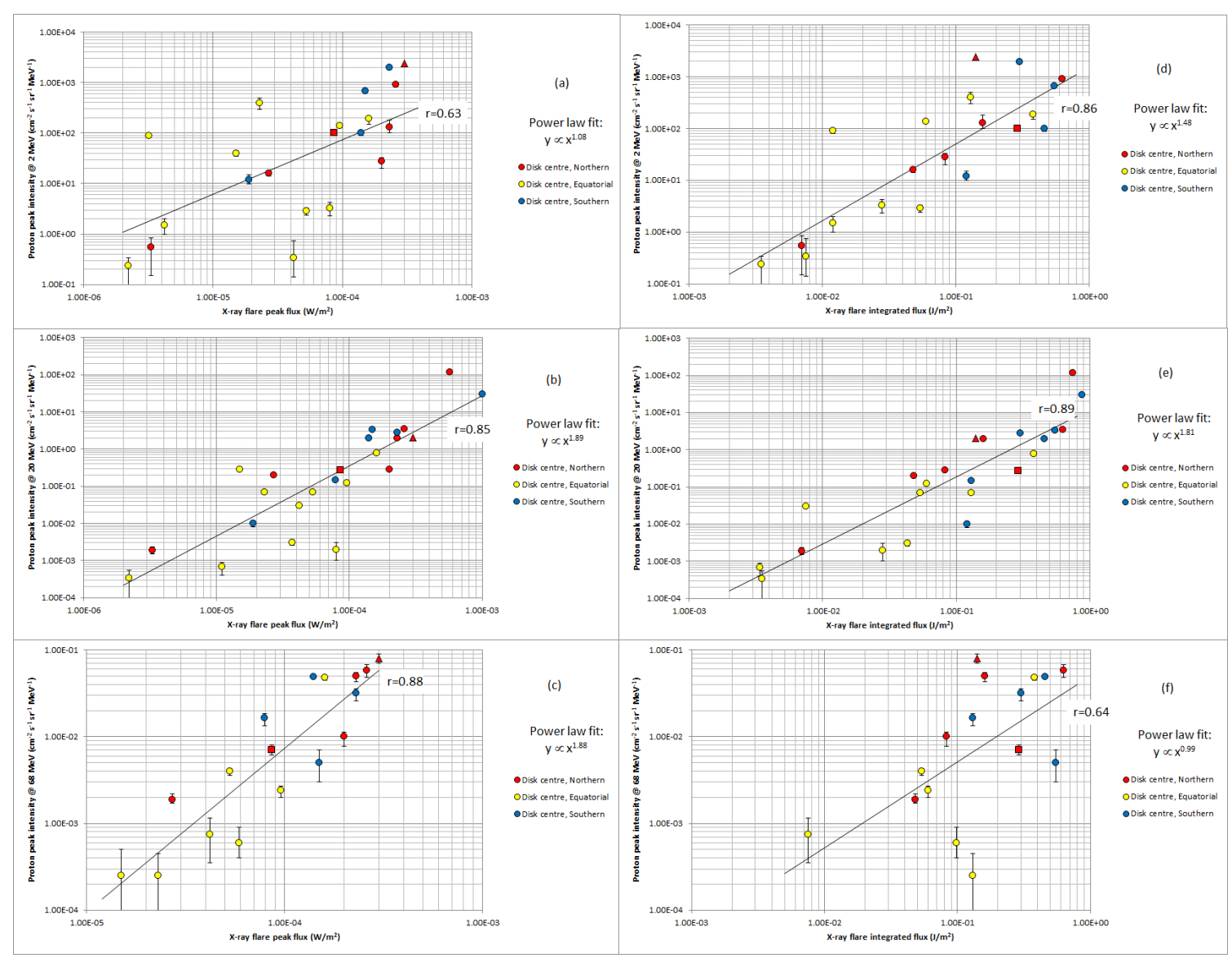

Figure 4: Proton peak intensities at 2, 20, and $68 \mathrm{MeV}$ as function of X-ray flare peak flux (a-c) and event integrated flux (e-f).

than for the projected (sky-plane) speed. We found no significant difference in the dependence of the peak intensities on the sky-plane or the space speed. Even for our restricted data set the variation of the peak intensity is still about two orders of magnitude at similar speeds. Not included in the correlation analysis, but shown in Figure 3 are also an event that started on July 16, 2002 and the first event of January 15, 2005. As mentioned before, the list [6] includes an event on July 15, 2002, which is associated with a CME at 20:45 UT. SOHO/LASCO halo CME catalog reports an event on July 15, 2002 at 20:30 UT, which is in our list of northern events (N15, W01). However, we could not find any enhancement of proton intensities close in time to this CME, but there was a clear, fast rising event the next day at $\sim 08: 00$ UT without any obvious source (CME or flare). This event on July 16, 2002 is plotted in Figure 3 as a red triangle. On January 15, 2005 two halo CMEs were observed by LASCO, the first one at 06:30 UT and the second at 23:06 UT. This second event is in our list of proton associated halo CMEs, but the first one was rejected, because when taking into account the solar B0 angle this event took place at latitude N21, just outside of our defined range. This event, however, is plotted as a red square in Figure 3.

Power law fits of proton peak intensities vs. CME speeds (linear fits in log-log scales) are presented in Figure 3. At the two lowest energies there is the trend of increasing power law index 
with increasing energy. However, the highest energy does not follow this trend and has a large scatter of the peak intensities. The power law index is larger at all energies when the peak intensities are plotted as a function of the space speed, indicating that the correction of the sky-plane speeds affects relatively more the lower speeds.

Proton peak intensities at 2,20, and $68 \mathrm{MeV}$ as function of the X-ray flare peak flux and event integrated flux are presented in Figure 4 on log-log scales. In the case of the X-ray peak flux, the correlation between the logarithms of the quantities is improving with increasing proton energy reaching a high value of 0.88 at $68 \mathrm{MeV}$. At the two lower energies, the correlation of the proton peak intensities with the event integrated X-ray flux is even better, but again at the highest energy the scatter of intensities is large with correspondingly weaker correlation with X-ray integrated flux. The power law indices of the fits of proton peak intensities vs. X-ray flare peak flux at 2 and $20 \mathrm{MeV}$ (1.89 and 1.88, respectively) are comparable with the results of [19] (1.59) and [20] (1.82), but in both these cases, with larger and less restricted data sets, much lower correlation coefficients (0.52 and 0.38, respectively) were obtained in[19] and [20].

\section{Conclusions}

We have investigated the association of solar proton events during the years 1996 to 2014 with full halo CMEs originating from near the disk centre (between longitudes E10 and W10) and the dependence of the proton peak intensities at energies 2, 20, and $68 \mathrm{MeV}$ on the CME speed and the magnitude of the associated soft X-ray flares. Altogether 64 full halo CMEs were selected from the SOHO/LASCO halo CME catalog, 26 from the equatorial [N10,S10] region, 16 northern events $((\mathrm{N} 10, \mathrm{~N} 20])$ and 22 southern events ((S10,S20]). These CMEs were associated with 25 proton events, and since no immediate difference between the proton events from various latitude regions was found, all three groups were combined in further analysis to maintain reasonable statistics.

A good correlation was found of the proton peak intensities with both the CME speeds and the X-ray flare magnitudes. The highest correlation with the CME speed was obtained for the $20 \mathrm{MeV}$ peak intensity vs. CME space speed $(r=0.83)$. Similarly for the X-ray flare magnitude, the highest correlation $(r=0.89)$ of the proton peak intensity with the integrated X-ray flare flux was found at $20 \mathrm{MeV}$. Even in the cases of the best correlations, the spread of the peak intensities at similar CME speeds or X-ray flare magnitudes is still roughly two orders of magnitude.

\section{Acknowledgments}

The SOHO/LASCO CME catalog is generated and maintained at the CDAW Data Center by NASA and the Catholic University of America in cooperation with the Naval Research Laboratory. SOHO is a project of international cooperation between ESA and NASA.

\section{References}

[1] N. Gopalswamy, S. Yashiro, S. Akiyama, P. Mäkelä, H. Xie, M.L. Kaiser, R.A. Howard, and J.L. Bougeret, Coronal mass ejections, type II radio bursts, and solar energetic particle events in the SOHO era, Ann. Geophys. 26, 3033, 2008. 
[2] D.V. Reames, Particle acceleration by CME-driven shock waves, AIP Conf. Proc. 516, 289, 2000.

[3] N. Gopalswamy, S. Yashiro, A. Lara, M.L. Kaiser, B.J. Thompson, P.T. Gallagher, and R.A. Howard, Large solar energetic particle events of cycle 23: A global view, Geophys. Res. Lett 30, 8015, 2003.

[4] N. Gopalswamy, S. Yashiro, S. Krucker, G. Stenborg, and R.A. Howard, Intensity variation of large solar energetic particle events associated with coronal mass ejections, J. Geophys. Res. 109, A12105, 2004.

[5] J. Hwang, K.-S. Cho, Y.-J. Moon, R.-S. Kim, and Y.-D. Park, Solar proton events during the solar cycle 23 and their association with CME parameters, Acta Astron. 67, 353, 2010.

[6] H.V. Cane, I.G. Richardson, and T.T. von Rosenvinge, A study of solar energetic particle events of 1997-2006: Their composition and associations, J. Geophys. Res. 115, A08101, 2010.

[7] J. Park, Y.-J. Moon, D.H. Lee, and S. Youn, Dependence of solar proton events on their associated activities: Flare parameters, J. Geophys. Res. 115, A10105, 2010.

[8] R. Miteva, K.-L. Klein, O. Malandraki, and G. Dorrian, Solar energetic particle events in the 23rd solar cycle: Interplanetary magnetic field configuration and statistical relationship with flares and CMEs, Solar Phys. 282, 579, 2013.

[9] S.W. Kahler and A. Vourlidas, Do interacting coronal mass ejections play a role in solar energetic particle events?, Astrophys. J. 784:47, 2014.

[10] S.W. Kahler and A. Vourlidas, Solar energetic particle events in different types of solar wind, Astrophys. J. 791:4, 2014.

[11] J. Park, Y.-J. Moon, and N. Gopalswamy, Dependence of solar proton events on their associated activities: Coronal mass ejection parameters, J. Geophys. Res. 117, A08108, 2012.

[12] S.W. Kahler, The correlation between solar energetic particle peak intensities and speeds of coronal mass ejections: Effects of ambient particle intensities and energy spectra, J. Geophys. Res. 106, 20947, 2001.

[13] G.E. Brueckner, R.A. Howard, M.J. Koomen et al., The large angle spectroscopic coronagraph (LASCO), Solar Phys. 162, 357, 1995.

[14] N. Gopalswamy, S. Yashiro, G. Michalek, H. Xie, P. Mäkelä, A. Vourlidas, and R.A. Howard, A catalog of halo coronal mass ejections from SOHO, Sun and Geosph. 5, 7, 2010.

[15] J. Torsti, E. Valtonen, M. Lumme et al., Energetic particle experiment ERNE, Solar Phys. 162, 505, 1995.

[16] E.W. Cliver and A.G. Ling, Electrons and protons in solar energetic particle events, Astrophys. J. 658, 1349, 2007.

[17] I.G. Richardson, T.T. von Rosenvinge, H.V. Cane, et al., >25 MeV proton events observed by the High Energy Telescopes on the STEREO A and B spacecraft and/or at Earth during the first $\sim$ seven years of the STEREO mission, Solar Phys. 289, 3059, 2014.

[18] E.W. Cliver, S.W. Kahler, and D.V. Reames, Coronal shocks and solar energetic proton events, Astrophys. J. 605, 902, 2004.

[19] E.W. Cliver, A.G. Ling, A. Belov, and S. Yashiro, Size distributions of solar flares and solar energetic particle events, Astrophys. J. 756, L29, 2012.

[20] J. Park and Y.J. Moon, What flare and CME parameters control the occurrence of solar proton events?, J. Geophys. Res. 119, 9456, 2014. 